\title{
"Business Continuity and Information Security Maintenance" Masters' Training Program
}

\author{
Natalia Miloslavskaya, Mikhail Senatorov, Alexandr Tolstoy, \\ and Sergei Zapechnikov \\ National Research Nuclear University MEPhI, \\ 31 Kashirskoe Shosse, 115409, Moscow, Russia \\ \{NGMiloslavskaya, MJSenatorov, tolstoy, SVZapechnikov\} @mephi.ru
}

\begin{abstract}
The experience of preparing for the "Business Continuity and Information Security Maintenance" (BC\&ISM) Masters' program implementation and realization at the "Information Security of Banking Systems" Department of the National Research Nuclear University MEPhI (NRNU MEPhI, Moscow, Russia) is presented. Justification of the educational direction choice for BC\&ISM professionals is given. The model of IS Master being trained on this program is described. The curriculum is presented.
\end{abstract}

Keywords: Information Security, Business Continuity, Standard, Model, Curriculum, Bachelor, Master, Specialist, Higher Education, Master's Program.

\section{Introduction}

In current conditions the critical infrastructure companies are faced with the challenges of their activities disruption or interruption. The reasons differ significantly: disruptions in an energy supply and services delivery, dysfunction of the information and telecommunication systems, presence of the information security (IS) threats in their information sphere and intentional actions or errors of their personnel, resignation of their key personnel, as well as fires, floods, man-made disasters etc.

The "business continuity" (BC) term has appeared in the second half of the 90ties. It was used in connection with the uninterrupted operation of a whole company, including first of all its main (key) and auxiliary business processes.

The BC maintenance process is to ensure the restoration of the company's functioning in the event of any unexpected or undesirable incident that could negatively affect the continuity of the critical business functions and their supporting elements, with a threat of losing the entire business.

In the framework of this process it is important for every company to address issues of IS maintenance. Accidentally the best practices experience shows (e.g., ISO/IEC 27002) that IS maintenance serves a common goal of business development and ensuring its continuity, since the information assets protection against various types of the IS threats can minimize the IS risks and maximize ROI (Return On Investment). 
The IS management international standards' (27000 series) analysis has allowed to justify the urgency of the BC\&ISM training program. Therefore it becomes evident at present that for the success of any business it is necessary to have knowledge in two interconnected areas - BC and IS. Thus the professionals with integrated knowledge, skills and abilities in these areas are in more demand than ever. That is why relevant staffing for the stakeholders is a very actual problem.

Analysis of the training programs, implemented by the various educational institutions of Russia and related to BC and IS, shows that a substantial dissatisfaction of the labor market needs in the professionals of this profile exists. That fact can be explained by the following reasons. Firstly, BC and IS maintenance (BC\&ISM) refers to a fairly new area of a professional activity. Secondly, the dynamics of this area is such that the existing Russian educational system with its high inertia lags behind the labor market needs.

The situation is complicated by the fact that the national system of higher education has lack of BC\&ISM professionals' comprehensive training. The BC issues are completely absent in the curricula of the existing IS training.

Partial satisfaction of the labor market needs is met at the level of an additional vocational training via implementation mainly of a few improvement courses and certification programs being implemented with an assistance of the BSI, the Disaster Recovery Institute, the BCM Institute etc. [1-3].

The article presents the experience of preparing for the implementation and realization of the "Business continuity and information security maintenance" (BC\&ISM) Masters' program at the "Information Security of Banking Systems" Department of the National Research Nuclear University MEPhI (NRNU MEPhI, Moscow, Russia).

\section{Choice of Educational Direction for BC\&ISM Professional Training}

The national system of higher education in Russia includes the following educational levels:

1) Bachelor (4 years' full-time training period, on the basis of the school education);

2) Master (2 years, continuing education after "Bachelor" qualification or the second higher education);

3) Specialist (5-5,5 years, on the basis of the school education).

There are two lists:

- a list of the training directions related to the implementation of the various profiles of bachelors and the various programs for masters;

- a list of the specialties.

For each training direction and each speciality there is the developed and approved basic national Federal state educational standard (with abbreviation FGOS from the Russian title of the standard) and corresponding approximate (so-called recommended) curriculum. 
Taking into account the above-mentioned features of the professional activities related to BC\&ISM, it is possible to consider the applicability of a particular level of education for the professionals training.

Firstly, a long-term training on the basis of higher education (bachelors, masters and specialists) cannot provide the required adaptation of the training content to the dynamically changing field of the graduates' professional activity.

Secondly, the strict framework for the training content, set by FGOS for bachelors and specialists, does not allow to take into account adequately the companies specifics under which BC\&IS challenges are addressed and where the graduates of the educational institutions will work.

Thirdly, among the training directions and specialities there is no FGOS directly related to BC\&ISM.

To our opinion the most appropriate way of addressing this challenge is to select an educational direction related to Masters' training. This approach has the following advantages:

- $\quad$ short training time (only 2 years);

- variability of the curriculum formation (up to $50 \%$ of training time can be given to the subjects, developed by an educational institution itself and directly related to the chosen field of their graduates' professional activity);

- flexibility of the curriculum implementation (up to15\% of training time can be given to so-called "optional (selected by the students themselves from the proposed set, at their own choice) disciplines" when the students choose to study the particular subjects, reflecting the specific objects of their professional activity, with which they may work during their further employment);

- practical orientation of training (up to $25 \%$ of training time can be given to the different types of practices (practical work), the implementation of their scientific and research works and preparation of a final qualifying work - PhD dissertation);

- curriculum adaptability (an educational institution must provide the students with a real opportunity to participate in their training program development themselves, including possible development of their individual educational programs).

The outlined above features enable to make a reasonable choice of the level of higher education "Master" as a base for the BC\&ISM professionals' training.

The Master's program development involves its binding to a specific educational direction. BC management in the presence of the IS threats in the information sphere relates directly to IS management. Therefore it is expedient to choose the 090900 "Information Security" educational direction and the corresponding FGOS [4].

\section{Model of a Graduate Trained on BC\&ISM Master's Program}

The model of a Master, trained on the BC\&ISM program, should define the areas, objects and types of a graduate professional activity and also the professional tasks, which he/she can solve in a company, where he/she will work after graduation. 
The modern Russian education is based on the so-called competence approach: the training outcome is stated as a list of the professional competencies that a student must acquire and that will determine its ability to apply his/her knowledge, skills and personal qualities to be successful in a particular area. Therefore the model should be supplemented by the appropriate professional competencies.

It should be noted that FGOS [4] contains the definition of the areas, objects and types of the professional activities, and also the professional tasks and the corresponding professional competencies related to basic IS Masters' training. These definitions were taken as a basis for describing the Master's model, adjusting them to a specific subject area of BC\&ISM.

The professional activity's area for the BC\&ISM Master's program graduates is the following: the areas of science, engineering and technology, covering the wide range of problems related to ensuring BC\&IS of the key company's business processes in conditions of presence of the IS threats in the information sphere.

The professional activity's object for the BC\&ISM Master's program graduates are the following:

- information resources and IT; computer, automation, telecommunication, information and analytical systems that ensure the key company's business processes;

- technologies ensuring BC\&IS of the key company's business processes;

- methods and tools for designing, modeling and experimental testing of systems, ensuring BC\&IS of the key company's business processes;

- management processes for ensuring BC\&IS of the key company's business processes.

The professional activity's types for the BC\&ISM Master's program graduates are the following:

- $\quad$ organizational and managerial;

- design;

- control and analytical;

- $\quad$ scientific and research;

- $\quad$ scientific and pedagogical.

The professional activity's types are related directly to a particular company's activities. At present the following companies' types that may need the BC\&ISM professionals can be outlined:

1) companies that create and operate systems ensuring BC\&IS of their business processes (such as banks);

2) companies developing such a systems (for example, system integrators);

3) companies engaged in control for assessing BC\&ISM level of the key company's business processes (for example, auditors);

4) companies carrying out scientific research and providing education (such as scientific and research centers and educational institutions). 
The professional activity's tasks for the BC\&ISM Master's program graduates are the following:

1). BC\&ISM systems' creation and operation relate to an organizational and managerial activity. Company's personnel are involved in addressing the following professional activity's tasks:

- participation in the projects on creating the BC\&ISM systems for the key company's business processes;

- preparing some methodical and legal documents' drafts, proposals and activity for the BC\&ISM systems for the key company's business processes;

- organization and implementation of a control activity for effectiveness assessment of the BC\&ISM systems for the key company's business processes;

- $\quad$ organization of an activity on improvement, modernization and unification of the BC\&ISM systems for the key company's business processes in compliance with the legal documents and requirements.

2). The BC\&ISM system for the business processes development relates to a design activity of the company's personnel and includes addressing the following professional activity's tasks:

- system analysis of an application area, identification of the IS threats and vulnerabilities in the company's information systems, development of the BC\&ISM requirements and criteria for the key company's business processes;

- conceptual design of the complicated systems, tool sets and technologies for BC\&ISM for the key company's business processes;

- justification of a choice of the functional structure, organizational principles for hardware, software and information management of the systems, tools and technologies for BC\&ISM for the key company's business processes;

- development of the BC\&ISM systems and technologies for the key company's business processes;

- adapting of the modern BC\&ISM techniques for the key company's business processes to a particular company on the basis of the domestic and international standards.

3). Implementation of the control functions on an assessment of the BC\&ISM level for the key company's business processes is related to a control and analytical activity, addressing the following professional activity's tasks:

- development of the control and analysis activity's programs;

- effectiveness control of the measures being used for BC\&ISM of the key company's business processes;

- implementation of the auditing programs for BC\&ISM of the key company's business processes;

- composing of a control and analytical activity's documentation.

4). In carrying out the scientific research and educational activities the universal tasks listed in FGOS are addressed [4].

The BC\&ISM Master's program is aimed at developing the graduates' specific professional competencies. At the end of the training they should be able: 
- $\quad$ to analyze and explore the BC\&ISM models and systems;

- to use in practice the BC\&ISM standards;

- $\quad$ to analyze the IS risks in order to ensure BC;

- to conduct synthesis and analysis of the design decisions on a company's BC\&ISM;

- to ensure the effective application of a company's information and technology resources to meet the BC\&ISM requirements;

- to participate in design and operation of a company's IS incident management;

- $\quad$ to participate in design and operation of a company's BC\&ISM systems;

- $\quad$ to conduct an instrumental IS monitoring in a company;

- $\quad$ to develop the proposals for a company's BC\&ISM systems;

- to develop and effectively implement a complex of the BC\&ISM measures (including rules, procedures, practical techniques, methods, guidelines, tools).

These professional competencies are established during a particular curriculum implementation.

\section{BC\&ISM Master's Program Curriculum}

The BC\&ISM Master's program curriculum (Table 1) has been created in 2011. It is a list of the disciplines with their labor content in credits and academic hours. All the disciplines are grouped in two training cycles:

- $\quad$ M1 - general scientific;

- $\mathrm{M} 2$ - professional.

Each training cycle has a basic part with a list of the disciplines determined by FGOS [4] and a variable part with the disciplines, identified by each educational institution itself and reflect the features of a particular Master's program.

The successful development of the professional competencies during the BC\&ISM Masters' training is impossible apart from the specific objects. Therefore in each variable part there are some optional disciplines, reflecting the specifics of such an objects. In the given curriculum (Table 1) each optional discipline matches two disciplines related to the Russian credit and financial sector (e.g. banks) and to the Russian nuclear industry (corresponding to the specifics of the NRNU MEPhI). During training within each optional discipline a student studies one of these disciplines considering a company's specifics, in which he/she will work after the graduation.

With the development of the Master's program the number of the disciplines related to a specific optional discipline can be increased, thereby extending the range of the professional activity's objects.

The curriculum has another two cycles that determine the time required and the labor content of the practice (internship), scientific research works (M3) and final state certification (M4). This part of the curriculum involves the students into implementation of some specific projects related to the BC\&ISM systems of a particular company's business processes.

This allows to create graduates' practical skills to apply the existing regulatory framework, to implement the IS risks (related to disasters) analysis, to establish the 
proper criteria for evaluating the possibility of an IS incident transformation into a disaster, to determine the IS related disaster scenarios, to choose the strategies and tools to ensure IS during the disaster and to develop the coordinated BC\&ISM programs.

Table 1. The BC\&ISM Master's Program Curriculum

\begin{tabular}{|c|c|c|c|}
\hline \multirow{2}{*}{ № } & \multirow{2}{*}{ Cycles, modules and disciplines } & \multicolumn{2}{|c|}{ Labor content } \\
\hline & & Credits & Hours \\
\hline \multirow{3}{*}{ M1 } & General scientific cycle (FGOS) & 24 & 864 \\
\hline & Basic part (FGOS) & 8 & 288 \\
\hline & Humanitarian module (FGOS) & 3 & 108 \\
\hline \multirow[t]{2}{*}{ M1.B1 } & Economics and Management. Part 1 (Economics) & 3 & 108 \\
\hline & Mathematical and natural sciences (FGOS) & 5 & 180 \\
\hline M1.B2 & Special sections of mathematics & 3 & 108 \\
\hline M1.B3 & Special sections of physics & 2 & 72 \\
\hline \multirow[t]{2}{*}{ M1.V } & Variable part (program) & 16 & 576 \\
\hline & Humanitarian module & 2 & 72 \\
\hline \multirow[t]{2}{*}{ M1.V1 } & Economics and Management. Part 1 (Management) & 2 & 72 \\
\hline & Mathematical and natural sciences & 8 & 288 \\
\hline M1.V & System analysis and system engineering & 2 & 72 \\
\hline M1.V & Applied theory of reliability & 2 & 72 \\
\hline M1.V & Decision making & 2 & 72 \\
\hline M1.V & Knowledge-based analysis of data and processes & 2 & 72 \\
\hline M1.VO & Optional Disciplines & 6 & 216 \\
\hline M1.VO1 & Optional discipline 1: & 2 & 72 \\
\hline M1.VO1.1 & Psychology and Pedagogy & & \\
\hline M1.VO1.2 & Research and development management & & \\
\hline M1.VO2 & Optional discipline 2: & 2 & 72 \\
\hline M1.VO2.1 & Banking system of the Russian Federation & & \\
\hline M1.VO2.2 & Fundamentals of nuclear non-proliferation & & \\
\hline M1.VO3 & Optional discipline 3: & 2 & 72 \\
\hline M1.VO3.1 & Banking basics & & \\
\hline M1.VO3.2 & Fundamentals of nuclear technology & & \\
\hline M2 & Professional cycle & 35 & 1260 \\
\hline M2.B & Basic (general professional) part (FGOS): & 9 & 324 \\
\hline M2.B1 & Protected information systems & 3 & 108 \\
\hline M2.B2 & Objects' IS maintenance technologies & 3 & 108 \\
\hline M2.B3 & IS management & 3 & 108 \\
\hline M2.V & Variable part (program) & 26 & 936 \\
\hline M2.V1 & Computer systems' security & 5 & 180 \\
\hline M2.V2 & BC\&ISM basics & 3 & 108 \\
\hline $\mathrm{M} 2 . \mathrm{V} 3$ & IS incident management basics & 2 & 72 \\
\hline M2.V4 & IS risks management basics & 2 & 72 \\
\hline M2.V5 & Information systems' disaster recovery & 2 & 72 \\
\hline M2.V6 & IT security assessment & 2 & 72 \\
\hline M2.V7 & $\mathrm{BC}$ maintenance management & 2 & 72 \\
\hline M2.VO & Optional Disciplines & 8 & 360 \\
\hline M2.VO1 & Optional discipline 1: & 2 & 72 \\
\hline M2.VO1.1 & IS of credit and financial sector's companies & & \\
\hline M2.VO1.2 & IS of nuclear objects & & \\
\hline
\end{tabular}


Table 1. (continued)

\begin{tabular}{|c|c|c|c|}
\hline \multirow{2}{*}{ № } & \multirow{2}{*}{ Cycles, modules and disciplines } & \multicolumn{2}{|c|}{ Labor content } \\
\hline & & Credits & Hours \\
\hline M2.VO2 & Optional discipline 2: & 3 & 108 \\
\hline M2.VO2.1 & Fundamentals of bank cards' security maintenance & & \\
\hline M2.VO2.2 & Physical security of nuclear objects & & \\
\hline $\mathrm{M} 2 . \mathrm{VO}$ & Optional discipline 3: & 3 & 108 \\
\hline M2.VO3.1 & Electronic documents interchange security & & \\
\hline M2.VO3.2 & Safety criteria and risk assessment & & \\
\hline M3 & Practice and Scientific Research Works & 50 & 1800 \\
\hline M4 & Final State Certification & 11 & 396 \\
\hline & Totally & 120 & 4320 \\
\hline
\end{tabular}

\section{Conclusion}

The IS management international standards' analysis has allowed to justify the urgency of the BC\&ISM training program.

The experience of preparing for BC\&ISM program implementation and realization at the "Information Security of Banking Systems" Department of the NRNU MEPhI shows that this program is more suitable to implement for Masters. Justification of the educational direction choice for the BC\&ISM professionals is given.

The BC\&ISM Master's program is designed for the applicants with the "Bachelor" qualification (for continuation of their higher professional education) or "Specialist" qualification (for the second higher education).

The model of IS Master being trained on this program is described. The model defines the areas, objects, types and tasks of a graduate professional activity.

The presented curriculum has been launched in the NRNU MEPhI from 2012. The first group of Masters (15 students) has started their training from fall 2012 and they still have not finished their first year.

Our findings may be useful for the tutors and managers of the similar training programs.

\section{References}

1. http://www.bsigroup.com/en-GB/iso-22301-businesscontinuity/iso-22301-training-courses

2. https://www.drii.org/education/education.php

3. http://www.bcm-institute.org/bcmi10/en/education

4. http://www.edu.ru/db-mon/mo/Data/d_09/prm497-1.pdf (in Russian) 